\title{
Former aux compétences interculturelles en école d'ingénieur
}

\section{Marc Weisser}

\section{(2) OpenEdition \\ Journals}

Édition électronique

URL : http://journals.openedition.org/ripes/997

ISSN : 2076-8427

Éditeur

Association internationale de pédagogie universitaire

Référence électronique

Marc Weisser, «Former aux compétences interculturelles en école d'ingénieur », Revue internationale de pédagogie de l'enseignement supérieur [En ligne], 31(3) | 2015, mis en ligne le 01 décembre 2015, consulté le 08 septembre 2020. URL : http://journals.openedition.org/ripes/997

Ce document a été généré automatiquement le 8 septembre 2020

Article L.111-1 du Code de la propriété intellectuelle. 


\title{
Former aux compétences interculturelles en école d'ingénieur
}

\author{
Marc Weisser
}

\section{Introduction}

1 Le but de cet article ${ }^{1}$ est de contribuer à la réflexion sur la conception de formations d'ingénieurs, en proposant un référentiel de compétences pour l'apprentissage de l'interculturalité et en suggérant deux approches pédagogiques compatibles avec l'approche par compétences. Après avoir argumenté la pertinence de notre focalisation, nous revenons successivement, et toujours d'un point de vue professionnel, sur la notion de culture et sur les composantes des compétences interculturelles. Nous croisons ensuite ces bases théoriques avec les référentiels de formation de la Commission des titres d'ingénieurs (CTI) et du Conceiving - Designing - Implementing Operating (CDIO). Ceci nous permettra finalement de questionner les pratiques pédagogiques pertinentes et de repérer les contraintes et opportunités qui caractérisent ce type de formation.

\section{Nécessité des compétences interculturelles dans les formations d'ingénieurs}

2 Les compétences interculturelles d'un individu visent à le libérer de ses modes de raisonnement habituels (UNESCO, 2013) pour qu'il puisse vivre les relations sociales dans des contextes culturels autres que le sien (Bartel-Radic, 2009). Pour le Conseil de l'Europe (2008), l'un des rôles majeurs des établissements d'enseignement supérieur réside dans le renforcement du dialogue interculturel : « l'Université se définit le mieux par son universalité ", à travers la formation d'« intellectuels interculturels " aptes à prendre toute leur place dans la société civile (p. 33). La profession d'ingénieur nous semble répondre tout particulièrement à ces intentions. En effet, leurs fonctions gestion de production, commercialisation, recherche et développement - les mettent 
sans cesse en contact avec des interlocuteurs appartenant à des classes sociales, à des aires géographiques ou encore à des groupes ethniques différents des leurs. Or, ces variations culturelles créent une distorsion dans la communication interpersonnelle (Bartel-Radic, 2009) en ce sens que chaque récepteur va interpréter les messages reçus en fonction de ses grilles de lecture particulières, de ses propres habitudes de décodage, de sa représentation personnelle de ce qu'est un échange, etc. Nous nous limiterons dans ce qui suit au cas des échanges linguistiques. Dans la compréhension des intentions d'autrui se trouvent ainsi mêlés le statut que nous lui reconnaissons, l'identité sociale dont il se réclame (Byram, Gribkova \& Starkey, 2002), nous rappelant la dimension performative de toute culture, qui permet de poser des actes rien qu'en s'exprimant (Lavanchy, Gajardo \& Dervin, 2011) à l'aide par exemple des éléments qu'on en extrait pour alimenter une stratégie de communication, pour assurer symboliquement une place déterminée d'interlocuteur.

3 Dans ces situations, il leur incombe alors de gérer des moments où des individus tendent à se conformer ou au contraire à transgresser certaines de ces normes culturelles, qu'ils utilisent " pour Dire et Se dire » (Abdallah-Pretceille \& Porcher, 1996, p.73). Leurs compétences interculturelles interviennent dans ce cas comme des « compétences sociales en contexte interculturel» (Bartel-Radic, 2009, p. 13) et leur permettent de dépasser une lecture ethnocentrée des événements auxquels ils sont confrontés. La dimension culturelle se superpose en effet aux habituelles dimensions interpersonnelles et institutionnelles en y important les croyances, les valeurs, les habitudes des uns et des autres. Se pose alors la question de savoir si les interactions aboutiront à la domination d'un groupe sur l'autre, ou à des attitudes défensives où chacun campe sur ses positions, ou encore à un métissage qui dépasse les clivages (Clanet, 1993).

4 L'amplification de ces phénomènes de nomadisme professionnel est prise en compte dans l'évolution des référentiels de langues par exemple : «Alors que le Niveau-seuil des années 70-80 constitue en catégorie spécifique "les spécialistes ou professionnels ne quittant pas le pays d'origine", une version actualisée de la société européenne, centrée sur la mobilité, doit réajuster la référence à cette situation, en valorisant l'action de ces professionnels sous l'angle de leurs compétences d'interface entre plusieurs systèmes de valeurs » (Zarate 2003, p. 97). Il s'agit alors de sortir de la juxtaposition de cultures hermétiques entre elles pour aller à une certaine porosité (Abdallah-Pretceille \& Porcher, 1996), à une ouverture à l'altérité, à une attention portée à ses raisons, à sa cohérence propre, sans pour autant forcément et immédiatement renoncer aux siennes.

5 Dans l'espace du Rhin supérieur où nous exerçons, trois régions, l'Alsace (France), le Land de Bade-Wurtemberg (Allemagne) et les cantons bâlois (Suisse), toutes de langue ou de dialecte germanique, se côtoient et échangent depuis toujours. Elles constituent à ce titre un bon exemple de ce que l'acculturation, entendue comme l'adoption par un groupe d'éléments culturels différents (Cuche, 1997), est la règle et non l'exception, de ce que les cultures sont d'abord métissées avant d'être particulières, du fait que vouloir les délimiter par des frontières est une illusion, qu'elles soient politiques, géographiques, sociales ou autres. Il est plutôt question de médiations, conçues comme espace de mise en relation des partenaires, ou comme régulation des situations de tension, ou encore comme dispositif créant sa dynamique propre par la confrontation à l'altérité (Zarate, 2003). 
6 Ces préalables relatifs au métier d'ingénieur à l'époque de la mondialisation des échanges étant posés, nous pouvons maintenant approfondir notre réflexion sur la notion de culture pour ensuite identifier les traits de compétences interculturelles à retenir pour les formations de ces futurs ingénieurs.

\section{Culture, interculturalité}

7 «La culture est l'ensemble des traits distinctifs, spirituels, matériels, intellectuels et psychologiques d'une société ou d'un groupe social, et englobe la totalité des manières d'être existant au sein d'une société ; elle comprend, au minimum, l'art et la littérature, les modes de vie, les manières de vivre ensemble, les systèmes de valeurs, les traditions et les croyances. Chaque culture est la somme des présupposés et des pratiques que partagent les membres d'un groupe et qui les distinguent d'autres groupes » (UNESCO, 2013, p. 10). Cette définition émanant d'un organisme international nous rappelle à la fois que c'est par la culture que l'homme s'adapte à la nature ou l'adapte à ses besoins, palliant ainsi une «formidable régression de ses instincts " (Cuche, 1996, p. 3), et que c'est par elle qu'il établit des distinctions, qu'il trie, classe et ordonne ce qu'il perçoit, ressent et pense (Abdallah-Pretceille \& Porcher, 1996).

C'est donc par notre culture que nous donnons sens à nos comportements et à ceux d'autrui, et que nous adaptons nos façons de faire à ce que nous estimons requis par nos valeurs ou attendu par nos interlocuteurs. On voit tout de suite là les risques inhérents aux situations interculturelles: incompréhension, quiproquo, déception ou surprise en outre lorsque nos interlocuteurs agissent de façon imprévue. Mais si la culture est " un ensemble de significations que se communiquent les individus d'un groupe donné à travers leurs interactions » (Cuche, 1996, p. 49), chacun d'entre nous dispose d'une marge de manœuvre qui lui laisse une certaine liberté interprétative, source d'individualisation des réactions, un espace d'évolution future aussi. La culture " peut donc être envisagée à la fois comme quelque chose d'institué, qui appartient à une "communauté" nationale, ethnique, religieuse ou autre, et comme un processus dynamique résultant de choix individuels » (Byram, 2009, p. 5). Ce dernier point nous évite de réifier les cultures : elles ne constituent pas des réalités objectives mais des discours, elles ne préexistent pas à leur énonciation en contexte (Abdallah-Pretceille \& Porcher, 1996).

9 Nous observons pour cette raison lors des rencontres interculturelles des processus de négociation du sens par lesquels, à tout le moins, chaque interlocuteur se voit mis en demeure d'entrer dans le jeu d'autrui, de comprendre ses raisons sans forcément les accepter, et par là de finalement mieux se connaître lui-même. Ce sont donc des situations d'apprentissage privilégiées, que l'on peut aisément relire à partir de l'assimilation et de l'accommodation piagétiennes: soit je «lis» le comportement des autres acteurs de la relation à partir de mes propres grilles, soit, pour entrer dans leur logique, je modifie mes schèmes culturels en me décentrant. L'ensemble requiert une attention accrue portée à l'image que l'on a de soi, que l'on voudrait donner de soi, que l'on pense avoir induite chez autrui. La tendance à la mono-identification constitue en cela un piège redoutable (Cuche, 1996): si pour moi mon interlocuteur n'est qu'un Allemand, ou qu'un technicien, ou qu'un latin, j'ai vite fait de lui prêter des valeurs, des façons de faire qui ne sont pas forcément les siennes; l'identification d'un individu à une seule culture l'enferme dans une image de lui-même, qu'il va soit rejeter parce 
qu'elle lui semble simplificatrice, stigmatisante, soit au contraire vouloir respecter avec un zèle excessif pour bien marquer sa différence. Dans les deux cas, les positions sont clivantes.

10 Nous préférons quant à nous placer les situations éducatives (formelles ou non) sous le signe de l'agir communicationnel (Habermas, 1981; voir par exemple Weisser \& Rémigy, 2005) qui suppose des échanges basés sur la réciprocité et sur l'égalité des interlocuteurs: elles participent ainsi à la construction de la compréhension personnelle par le biais d'une recherche mutuelle de l'intercompréhension. «Dans l'approche sémiotique [des phénomènes interculturels], la communication est considérée comme étant production et échange de signes. Les sémioticiens de l'interculturel observent comment les produits de cette communication interagissent avec les individus pour leur donner du sens. Ils proposent également que ces individus sont impliqués constamment dans la création et l'interprétation de signes " (Dervin, 2011, p. 31), à partir de leurs horizons d'attente (Gadamer, 1976), de structures d'anticipation enracinées dans leur tradition culturelle (Lavanchy et al., 2011). Plutôt que d'essayer de caractériser chaque culture par ses particularités « ou ses discours de vérité (chez moi, on fait comme ça; je suis biculturel...) » (Dervin, 2011, p. 112), il convient d'être attentif aux interactions langagières ou plus largement aux processus symboliques (incluant le non verbal) lorsque l'on veut étudier les phénomènes interculturels, mais aussi, dans une pédagogie des compétences interculturelles, de faire fonds sur les échanges sémiotiques, dialogiques, nous y reviendrons. Détaillons pour l'instant quelles pourraient être les composantes des compétences interculturelles pertinentes dans les formations pour ingénieurs, dont nous traiterons dans le cadre réglementaire français (attentes de la $\mathrm{CTI}$ ) mais dans une perspective résolument internationale (propositions de l'Organisation de Coopération et de Développement Économiques et de l'Union Européenne).

\section{Compétences interculturelles pour l'ingénieur}

11 «[Si] la pluriculturalité désigne la capacité à s'identifier et à participer à des cultures différentes, l'interculturalité désigne la capacité à faire l'expérience de l'altérité culturelle et à l'analyser, et à se servir de cette expérience pour réfléchir à des questions généralement considérées comme allant de soi au sein de sa propre culture ou de son milieu» (Byram, 2009, p.7). Nous avons ci-dessus essayé de lister les interlocuteurs de cultures différentes rencontrés par l'ingénieur dans l'exercice de sa profession, qu'il s'agisse de personnes appartenant à d'autres aires linguistiques, à d'autres catégories socioprofessionnelles, à d'autres "cultures d'entreprise», etc. Si maintenant nous choisissons d'entrer par ses tâches et par les capacités qu'elles requièrent, telles que décrites par la CTI (2009), nous remarquons que la définition de Byram (2009) peut s'appliquer à nombre d'entre elles : aptitude à prendre en compte les exigences commerciales, aptitude à prendre en compte les enjeux de relation au travail, d'éthique, capacité à animer une organisation et à la faire évoluer, engagement et leadership...2

12 Les compétences interculturelles reposent sur quelques principes de base : ouverture à l'altérité (curiosité, empathie), connaissance de soi (et évaluation de ses propres modèles), négociation entre ses croyances propres et celles d'autrui (sans vouloir s'identifier à l'Autre), compétence d'interaction et d'analyse (pour agir ensemble en 
vue d'objectifs communs) (Byram, 2009 ; Dervin, 2004 ; UNESCO, 2013). Elles font appel à cinq catégories de ressources :

- des savoir-être, comme l'aptitude à se décentrer, à réviser sa méfiance face à autrui ;

- des connaissances sur le fonctionnement des groupes sociaux, sur leurs valeurs ;

- un « savoir comprendre " visant à interpréter correctement des sources étrangères ;

- un savoir apprendre de nouvelles connaissances et savoir les mobiliser en temps réel en situation de communication ;

- et enfin un « savoir s'engager ", de niveau taxonomique ${ }^{3}$ plus élevé, puisqu'il se rapporte à

l'évaluation critériée, objectivée, des points de vue et pratiques endogènes et exogènes (Byram et al., 2002).

13 Cette description correspond bien au modèle général de la compétence retenu tant par les employeurs que par les formateurs. On prendra pour exemple des premiers la définition du Mouvement des entreprises de France (MEDEF, 1998) : «La compétence professionnelle est une combinaison de connaissances, savoir-faire, expériences et comportements, s'exerçant dans un contexte précis. Elle se constate lors de sa mise en œuvre en situation professionnelle à partir de laquelle elle est validable. C'est donc à l'entreprise qu'il appartient de la repérer, de l'évaluer, de la valider et de la faire évoluer " (s.p.). Scallon (2004) considère qu'une situation de compétence réclame la mobilisation d'une série de ressources qui relèvent du « savoir que », ou qui sont autant de savoir-faire, de savoir-être et d'autres comportements stratégiques. Dans tous les cas, nous avons affaire à un modèle composite qui tisse des relations réciproques entre des éléments de connaissances disciplinaires et des attitudes plus transversales à l'égard d'autrui ou des problèmes professionnels. L'approche par compétences (APC) pousse ainsi à un changement de perspective du point de vue de l'ingénierie de formation : les disciplines académiques ne sont plus premières et n'imposent plus leur logique d'exposition interne, mais n'en restent pas moins extrêmement importantes, tant chaque compétence est étayée des savoirs issus des sciences de la nature ou des sciences humaines (Chauvigné \& Coulet, 2010). Simplement, le choix des contenus disciplinaires est désormais assujetti au référentiel de compétences, on n'enseignera que ce qui est reconnu comme ressource d'une compétence donnée.

Cette approche est cependant nécessaire si l'on considère les nouvelles caractéristiques de l'entreprise: élargissement des tâches, réduction de la ligne hiérarchique, développement du caractère collectif du travail (Wittorski, 2012). La diversification des activités professionnelles et bien souvent leur gestion simultanée imposent à l'opérateur flexibilité, réactivité, adaptabilité. Dans ces conditions, la déclinaison d'une formation en référentiel de compétences est un facteur important de sa visibilité (Pentecouteau, 2012), et même de sa légitimité. Les équipes pédagogiques peuvent exploiter cette contrainte venue de l'extérieur comme un prétexte à renouveler leurs méthodes de formation (Balas \& Riem, 2014 ; Wittorski, 2012). Or, la CTI (2009), qui accrédite les projets de formation des écoles d'ingénieurs en France, laisse à ces écoles l'entière responsabilité de définir la liste des compétences sanctionnées par leurs diplômes. Elle présente l'ingénieur comme « un cadre scientifique et technique sachant de plus prendre en compte les enjeux industriels, sociétaux et environnementaux " (CTI, 2009, p.6). Le syllabus de la CDIO (2001) va dans le même sens: parmi les compétences et les attitudes liées à la personne, elle identifie entre autres la persévérance et la flexibilité, la tolérance à la critique, l'envie de travailler avec autrui et de prendre en considération son point de vue, le respect des coutumes et normes 
autorisant les contacts interpersonnels à l'international, la communication interculturelle, etc. Tout ceci légitime non seulement notre projet d'intégrer les compétences interculturelles dans les formations d'ingénieurs, mais montre de plus qu'elles sont déjà considérées comme indispensables par des organismes d'évaluation de ces formations. Ceci nous permet d'avancer la proposition suivante, qui croise une sélection des compétences de l'ingénieur telles qu'explicitées par la CTI (2009, p. 27, en italiques ci-dessous) avec les cinq catégories de ressources des compétences interculturelles proposées par Byram et al. (2002) mentionnées précédemment, et qui décrit ce que le futur diplômé devra savoir mettre en œuvre en situation d'emploi :

C1 : «Capacité à s'intégrer dans une organisation, à l'animer et à la faire évoluer: connaissance de soi, esprit d'équipe, engagement et leadership."

- Savoir-être : être curieux envers les modes d'organisation des groupes humains, s'ouvrir à la « culture d'entreprise»;

- Savoirs : connaitre les relations entre catégories de personnels présents dans l'entreprise, connaître les modes de représentation et les dispositifs de dialogue dans l'entreprise ;

- Savoir comprendre : porter attention à la presse syndicale, aux comptes rendus du comité d'entreprise, etc.;

- Savoir apprendre / faire : exploiter un «stage ouvrier », participer au dialogue social ;

- Savoir s'engager : évaluer de façon critique des positions des différents groupes sociaux, décentration, mieux connaître ses propres façons de prendre position.

C2: "Aptitude à travailler en contexte international: maîtrise d'une ou plusieurs langues étrangères, ouverture culturelle, expérience internationale."

- Savoir-être : remarquer ettolérer l'ambiguïté dans les relations internationales, tirer profit d'une année sabbatique ou d'un semestre à l'étranger ;

- Savoirs : connaître les normes des relations hiérarchiques et des relations commerciales dans lesquelles se reconnaissent les partenaires;

- Savoir comprendre : maîtriser une ou plusieurs langues étrangères, jusqu'à l'identification des implicites du discours d'autrui ;

- Savoir apprendre / faire : s'informer sur les entreprises concurrentes à l'international, proposer des stratégies qui en tiennent compte ;

- Savoir s'engager : juger les pratiques des entreprises concurrentes à partir de critères économiques et sociaux, prendre conscience des priorités retenues par son propre employeur.

C3 : "Aptitude à mettre en cuvre les principes du développement durable: environnement, économie, social et gouvernance."

- Savoir-être : replacer les valeurs de l'entreprise dans une perspective historique, concevoir des projets viables à long terme dans une économie mondialisée ;

- Savoirs : connaître les conditions de production des matières premières, connaitre les formes de recyclage des produits en fin de vie dans une économie mondialisée ;

- Savoir comprendre : analyser les effets de l'économie (ou de telle industrie) sur la société et sur l'environnement dans une économie mondialisée, comparer les modèles de développement;

- Savoir apprendre / faire : tirer argument de ses savoirs pour infléchir la gouvernance de l'entreprise ;

- Savoir s'engager : utiliser des critères sociaux et écologiques en plus des critères économiques et financiers pour évaluer un mode de production industriel. 
C4: : Aptitude à prendre en compte et à faire respecter des valeurs sociétales : appropriation des valeurs sociales, de responsabilité, d'éthique, de sécurité et de santé."

-Savoir-être : suivre les débats sociaux concernant le système de production, distinguer et chercher à comprendre la diversité des réponses à ces questions ;

- Savoirs : identifier les valeurs invoquées par les différents groupes sociaux et les positionnements qu'elles induisent en conséquence;

- Savoir comprendre : comparer sa propre position à celle d'autrui, expliciter les raisons des uns et des autres;

- Savoir apprendre / faire : devancer les arguments de son interlocuteur dans la négociation commerciale ou sociale, communiquer de façon compréhensible et acceptable par son interlocuteur ;

- Savoir s'engager : estimer la compatibilité des choix de l'entreprise avec la diversité des valeurs et pratiques présentes dans le corps social, apprécier les effets du travail sur le sentiment d'appartenance des employés.

19 Cette façon de planifier l'apprentissage de compétences interculturelles peut bien entendu s'appliquer à d'autres éléments de référentiels de formation que les écoles d'ingénieurs retiendraient pour spécifier les acquis de leurs diplômés, selon leurs spécialités, selon le bassin d'emploi ou le type de fonctions visés.

Par ailleurs, soulignons que si la pédagogie par objectifs reposait sur une psychologie behavioriste, l'APC ne vise pas, quant à elle, à faire acquérir des comportements reproductibles, mais à « développer des dispositions à générer des conduites adaptées face à des situations diverses et changeantes » (Chauvigné \& Coulet, 2010, p. 16) dans une perspective désormais socioconstructiviste tout à fait compatible avec les attendus de la relation interculturelle. Cette dernière réclame en effet une capacité à la gestion des dysfonctionnements, à la résolution de familles de situations-problèmes, à la mobilisation de ressources différentes selon ce que nécessite le contexte de l'interaction en cours. "Sans doute, le moteur profond de l'interculturalité se trouve dans cette recherche d'une conjonction dynamique des formes de la culture, entre elles et avec les conduites d'action. La conjugaison affrontée de l'interculturel factuel [qui est subi, qui n'est perçu que quand il est source d'ambiguïté] et de l'interculturel volontaire [qui est voulu et qui parfois est paré de qualités souhaitables mais peu réalistes] est, peut-être, de cet ordre » (Demorgon, 2003, p. 65). L'ingénieur est de toute façon confronté à l'interculturel factuel, nous l'avons montré en préambule; les compétences dont nous visons l'acquisition lui permettront de passer à l'interculturel volontaire à travers sa construction identitaire (Wittorski, 2012) comme professionnel en contact avec des personnes culturellement éloignées, tant au plan de leur appartenance à un groupe social différent qu'à celui de la nationalité ou de la langue d'usage.

\section{Méthodes de formation aux compétences interculturelles}

21 Le choix de l'APC n'a pas que des répercussions au niveau du référentiel de formation, tel que développé dans l'exemple ci-dessus, mais aussi au niveau des méthodes de formation et des dispositifs d'évaluation (Balas \& Riem, 2014). Les pratiques pédagogiques vont s'en trouver modifiées, notamment du point de vue des rapports 
entre les acteurs en relation (Chauvigné \& Coulet, 2010). Nous avons invoqué ci-dessus la théorie de l'agir communicationnel de Habermas (2001), nous aimerions y revenir de façon plus détaillée. Notons pour commencer que la perception des rapports interindividuels défendue par Habermas (2001) est compatible à la fois avec la pédagogie socioconstructiviste requise par l'APC et avec les éléments définitoires que nous avons reconnus aux compétences interculturelles: "La compétence communicative [y] est considérée comme le pivot du développement de l'identité personnelle au sein de l'environnement social. [...] Pour qu'il y ait compétence communicative, il faut qu'au préalable quatre conditions soient réalisées dans le discours :

- la vérité des assertions ;

- l'adéquation des normes communes, des relations interpersonnelles (égalité de statut) et du contexte ;

- la véracité de la représentation de soi et

- le caractère compréhensible du contenu sémantique de l'énonciation » (Neuner, 2003, p. 61).

L'apprentissage de compétences interculturelles va de ce fait consister en une renégociation permanente de ces points dans la confrontation à un autrui différent. Clanet (1993) cite par exemple le «recadrage » comme procédé intéressant dans ce domaine, en ce qu'il consiste à changer de prémisses, à ne plus considérer que notre interprétation du monde est la seule recevable. S'il y a égalité reconnue des statuts des interlocuteurs, la mise en tension des traditions respectives va déboucher sur la conscientisation des préjugés de chacun, première étape vers leur prise en compte dans le processus de communication et, éventuellement, vers leur modification (Lavanchy et al., 2011).

Comme dans tout dispositif pédagogique, l'introduction d'un objet tiers évite de sombrer dans la confrontation directe des personnes. La médiation d'un contenu de savoir à maîtriser, ou d'un projet à réaliser ensemble, constitue une bonne façon de favoriser les apprentissages interculturels (Cazenave, 2003). Nous pouvons même estimer qu'ils s'en avèrent simplifiés, dépassionnés en quelque sorte. L'« intelligibilité des faits inscrits dans une structure caractérisée par la pluralité linguistique et culturelle» (Abdallah-Pretceille \& Porcher, 1996, p. 59) que recherche l'approche interculturelle se conquiert plus sereinement lorsque ces derniers sont produits en contexte, et en ce qui nous concerne dans le cadre de cet article, en contexte professionnalisant. Ces actions doivent en effet non seulement faire la preuve de leur efficacité, mais aussi de "leur légitimité au regard des us et coutumes du groupe professionnel » (Wittorski, 2012, p. 7) conçu ici comme ayant une culture propre avec des façons de faire jugées idoines.

À travers de tels dispositifs requérant des échanges entre individus aux identités distinctes, les formateurs seront attentifs aux « rapports fonctionnels qu'entretiennent la communication et la culture, non pas seulement au niveau du message, mais de la relation à l'Autre» (Abdallah-Pretceille \& Porcher, 1996, p. 61). La compétence interculturelle est au final surtout une compétence pragmatique, au sens linguistique du terme (Moeschler \& Reboul, 1994), une façon d'utiliser les systèmes de signes et le discours pour simultanément faire une place à autrui dans ses différences et justifier de sa position propre, relativement non pas à une sorte de bonne volonté allant de soi (interculturel volontaire) (Demorgon, 2003), mais à un projet à mener en commun avec 
une obligation de réussite. Ceci va, à terme mais de façon plus durable, amener l'étudiant à "restructurer le système d'attitudes qui, en chacun de nous, commande notre représentation d'autrui » (Camilleri, 1993, p. 44). Les théories de l'énonciation et les outils d'analyse qu'elles proposent deviennent alors des aides appréciables pour le formateur (Weisser, 2007) qui va chercher à observer puis à évaluer les "procédés linguistiques par lesquels le locuteur imprime sa marque à l'énoncé, s'inscrit dans le message (implicitement et explicitement) et se situe par rapport à lui » (Dervin, 2011, p.114). Les situations d'apprentissage vont devoir être aménagées pour que les apprenants gèrent collectivement leurs interactions et leurs tâches, puisque ce sont elles qui sont facteur d'apprentissage. On aura donc recours systématiquement à des travaux de groupes, si possible constitués d'individus aux identités contrastées. Le groupe est en effet à la fois un système intermédiaire entre l'individuel et le collectif, une espace dynamique où déséquilibres, tensions, adaptation et accommodation poussent chacun à évoluer en s'adaptant à autrui, un espace de structuration, à travers la prise de conscience des processus vécus en temps réel, gage de leur transférabilité ultérieure (Clanet, 1993). Dans les sous-sections ci-dessous, nous proposons deux exemples de méthodes de formation alliant travaux de groupe et apprentissage de compétences interculturelles, spécifiées à la formation des ingénieurs.

\subsection{Apprentissage par incidents critiques}

L'UNESCO (2013) suggère de « recueillir des exemples significatifs de cas dans lesquels le contexte culturel constitue un facteur clé » (p. 27), dans son optique pour l'exercice des droits et libertés de chacun. D'autres approches soulignent la même importance «d'accorder de l'attention non plus aux règles, aux structures, aux explications, mais de considérer les exceptions, les instabilités, les détournements » (Lavanchy et al., 2011, p. 28), en ce qu'un « conflit passé, reconnu parce que nommé, a plus de chance d'être dépassé qu'un conflit refoulé, et inscrit dans une mémoire honnie " (Zarate, 2003, p. 94). C'est donc par l'étude des dysfonctionnements - souvent regrettables mais parfois aussi heureux - plutôt que par l'identification des régularités que la compréhension d'autrui peut progresser, à travers l'explicitation des présupposés de chacun. Ceci valide la méthode des incidents critiques, à la fois dans une perspective de recherche mais aussi dans un but de formation (Stoiciu, 2008).

Nous appellerons incident critique un événement limité dans le temps, qui ne correspond pas à nos attentes immédiates, et suffisamment important dans ce qu'il révèle ou dans ce qu'il entraîne pour nécessiter une intervention réfléchie (Leclerc, Bourassa \& Filteau, 2010; Lescarbeau, 2000). En tant qu'outil pédagogique, la description de chacun d'entre eux comporte un bref tableau de la situation génératrice d'incompréhension et la succession des actions, paroles et éventuellement sentiments des protagonistes; elle n'explique par contre pas les différences culturelles, qui sont à induire lors de l'exercice à partir de l'analyse des comportements (Apedaile \& Schill, 2008). Dans l'idéal, l'ensemble de ces données gagne à être recueilli en situation réelle, par entretien avec les personnes concernées elles-mêmes, avec leurs propres mots, leurs propres grilles de lecture. Un moment propice peut être le débriefing lors d'un retour de stage par exemple. Comme le suggère la linguistique pragmatique, c'est ensuite à travers les interactions entre deux cadres de références culturelles (Body Consortium, 2013) que l'on va pouvoir expliciter les systèmes de normes, d'attentes mis en jeu par chacun, permettant ainsi de dépasser le choc interculturel. 
27 La démarche de formation en elle-même peut se décomposer en trois temps, «la décentration, pour mieux cerner sa propre identité socio-culturelle, la pénétration du système de référence de l'autre, et enfin la négociation et la médiation " (CohenEmérique, 1993, p. 76). La décentration permet de prendre du recul, de se percevoir comme un autrui ; c'est la confrontation à l'inattendu, au surprenant qui la rend possible. Nous retrouvons là la première des catégories de Byram et al. (2002), de l'ordre du savoir-être, présentée précédemment. La découverte de l'organisation mentale de l'interlocuteur repose ensuite à la fois sur les informations que nous avons acquises sur son cadre de vie et ses valeurs (connaissances) et sur la qualité de notre écoute, de notre attention portée aussi au non verbal (savoir comprendre). Les tentatives de résolution du conflit des interprétations reposent enfin sur notre évaluation de la situation (savoir s'engager) et en conséquence sur les décisions prises relativement à l'ampleur du compromis. Que nous dit le cadre règlementaire? Jusqu'où accepter d'aller à l'intérieur de celui-ci sans transiger sur certains principes? Dans l'idéal, la solution trouvée sera de type gagnant-gagnant; à tout le moins protégera-t-elle la face de chacun des protagonistes (Cohen-Emérique, 1993).

Les assimilateurs de culture (culture assimilator) systématisent ce procédé, chaque incident critique y étant accompagné de différents dénouements possibles à partir desquels la discussion entre apprenants s'engage (voir par exemple Apedaile \& Schill, 2008 ; Bartel-Radic, 2009 ; Korhonen, 2003). Leur inconvénient réside dans le fait qu'ils proposent des situations décontextualisées. Nous aimerions par conséquent réfléchir à la façon d'appliquer cette technique à notre référentiel de compétences interculturelles pour l'ingénieur tel que décrit précédemment. Reprenons la compétence C2 « Aptitude à travailler en contexte international: maîtrise d'une ou plusieurs langues étrangères, ouverture culturelle, expérience internationale » et les différentes ressources qu'elle mobilise. Le semestre passé à l'étranger peut avoir pour objectif pour l'élève-ingénieur, entre autres, de relever certaines situations marquées par l'ambiguïté, en les détaillant comme proposé ci-dessus (savoir-être). Au retour en école, une recherche documentaire sera faite pour identifier les normes des relations entre collègues et des relations commerciales dans lesquelles se reconnaissent les partenaires étrangers (savoirs). Simultanément, les cours de langue pourront être axés sur l'élucidation des implicites dans les discours professionnels, sur les nuances possibles relativement aux positions hiérarchiques dans l'entreprise grâce à différents procédés d'énonciation, sur le langage non verbal aussi: proxémique (occupation de l'espace autour de soi), kinésique (communication gestuelle) (savoir comprendre). Ce travail préparatoire aura permis le processus conjoint de connaissance de soi et de compréhension d'autrui (Cohen-Emérique, 1993). La dernière phase de ces études de cas consistera alors à juger des pratiques ainsi identifiées et lestées de leurs présupposés culturels (savoir s'engager) pour définir les stratégies de réponses industrielles ou commerciales facilitant l'accord entre les partenaires (savoir-faire).

\subsection{Apprentissage par résolution de problèmes}

La seconde méthode que nous désirons présenter, l'apprentissage par résolution de problèmes, est déjà fréquemment utilisée en école d'ingénieurs, mais plutôt pout la formation scientifique et technologique (Barge, 2010 ; Champagnol, 1974 ; Clark, 2009 ; Poirier Proulx, 1997 ; Romainville, 1999). Retenons qu'il s'agit d'une démarche relevant elle aussi du socioconstructivisme en ce qu'elle se base sur des travaux en petits 
groupes hétérogènes, qu'elle tient compte des représentations initiales des étudiants (l'apprentissage allostérique de Giordan \& De Vecchi (1987) s'appuie par exemple sur ces préconceptions pour les remettre en question et finalement les surmonter), et qu'elle propose des apprentissages significatifs parce que répondant à des problèmes professionnels. A la différence des exercices, des travaux pratiques et des autres études de cas, elle impose non pas le réinvestissement, le transfert de connaissances acquises par ailleurs, mais la découverte de nouvelles compétences pour, justement, résoudre un problème.

Elle peut s'organiser en une petite dizaine de phases que nous allons détailler et en même temps spécifier aux objectifs du présent texte, et plus particulièrement encore à la compétence $\mathrm{C} 2$ «Aptitude à mettre en cuvre les principes du développement durable: environnement, économie, social et gouvernance » et à ses ressources :

- choisir en contexte interculturel la situation problème à dénouer, relevant ici du développement durable. Elle peut concerner entre autres les conditions de production des matières premières, ou les formes de recyclage des produits en fin de vie ;

- identifier et clarifier les concepts peu familiers, s'accorder au sein du groupe sur les définitions des termes du problème telles qu'elles sont retenues par les organismes internationaux : empreinte carbone, soutenabilité, écoresponsabilité, commerce équitable, etc. (savoirs);

- définir le problème dans le cadre d'une économie mondialisée : thème général ? Buts visés ? Une composition de groupes mêlant des étudiants d'origines sociales ou géographiques diversifiées est souvent possible en école d'ingénieurs et tout à fait pertinente dans le présent dispositif ;

- analyser le problème et clarifier ses représentations initiales : ce que je sais, ce que je crois savoir, ce que je pense ne pas savoir... On insistera là sur l'explicitation des normes culturelles personnelles, des allants-de-soi de chacun. Les interactions entre des apprenants aux identités éloignées seront propices à ce travail de connaissance de soi ;

- effectuer l'inventaire des hypothèses à envisager, identifier les relations qu'elles entretiennent entre-elles. La curiosité, l'ouverture d'esprit et l'échange entre membres du groupe permettront de conjecturer de façon innovante, en sortant justement des cadres de référence individuels non questionnés ;

- formuler des objectifs d'apprentissage, un planning, et répartir les tâches dans le groupe ;

- effectuer des recherches : collecter, articuler, rédiger la synthèse en regard des hypothèses préalables. L'analyse des effets de l'économie (ou de telle industrie) sur la société et sur l'environnement dans une économie mondialisée peut déboucher sur une comparaison des modèles de développement (savoir comprendre) ;

- mettre en commun au sein de l'équipe, rediscuter les apports de chacun, par rapport aux valeurs de l'entreprise dans une perspective historique de développement (savoir-être);

- proposer une solution au problème et la transférer à des situations problèmes analogues de gouvernance d'entreprises (savoir-faire) ;

- s'autoévaluer dans un dernier temps métacognitif : ce que je sais de neuf, comment j'ai fait pour y arriver, seul et en groupe, ce que j'ai dû remettre en question pour y arriver, dans mes représentations initiales, dans mes savoirs, dans mes habitudes, dans mes valeurs (savoir s'engager). 


\section{Conclusion}

31 Les ingénieurs du $21^{\mathrm{e}}$ siècle devront plus que jamais être formés aux relations interculturelles dans le cadre d'une économie mondialisée ; leurs différentes missions le réclament, la variété de leurs interlocuteurs aussi. Ces nouvelles compétences les porteront vers des modèles de communication plus à l'écoute d'autrui dans ses différences, les rendront attentifs aux phénomènes d'ambiguïté, de compréhension autre ; elles leur permettront aussi d'opter pour des attitudes de plus grande tolérance à la surprise et pour une démarche de recherche éclairée de consensus dans des négociations professionnelles ou commerciales généralement situées en contexte interculturel.

Notre analyse a tenté de montrer que la formation des ingénieurs est finalement tout à fait prête à laisser une place de choix à la construction de compétences interculturelles. D'un côté, les recherches sur l'interculturalité s'accordent à reconnaitre quelques principes sur lesquels bâtir les échanges avec autrui, qui débouchent sur une série de ressources génériques à mobiliser en contexte. De l'autre, les référentiels nationaux (CTI) et internationaux (CDIO) de formation des ingénieurs proposent un cadre tout à fait compatible avec ces préceptes. C'est ce que nous avons essayé de concrétiser en croisant les deux approches à l'aide de dispositifs d'apprentissage investissant respectivement la méthode des incidents critiques puis l'apprentissage par résolution de problèmes. Cette façon de faire est transférable à d'autres situations et à d'autres compétences; elle peut également être encore spécifiée puisque nous n'avons travaillé qu'avec le référentiel indicatif de la CTI et non à partir de la formation à telle spécialité du métier d'ingénieur, dans un bassin d'emploi ou une branche professionnelle particuliers.

33 Au terme de notre réflexion, nous serions tenté de dire que finalement, on ne part pas le la culture pour comprendre l'acculturation, mais de l'acculturation pour comprendre la culture (Cuche, 1996). Aucune culture n'existe à l'état pur, et encore moins en contexte transfrontalier tel que nous le vivons localement. De plus, chacun d'entre nous (et de nos étudiants) appartient simultanément à plusieurs cultures (sociale, nationale, spécifique au genre, religieuse, etc.). L'acquisition de compétences interculturelles va alors permettre des échanges plus sereins avec un autrui différent, mais aussi et simultanément de mieux se connaître et se comprendre soi-même en tant que personne aux identités multiples (Byram et al., 2002).

\section{BIBLIOGRAPHIE}

Abdallah-Pretceille, M. \& Porcher, L. (1996). Éducation et communication interculturelle. Paris : Presses Universiaires de France.

Apedaile, S. \& Schill, L. (2008). Critical Incidents for Intercultural Communication. Edmonton : NorQuest College. 
Balas, S. \& Riem, P. (2014). Conception de référentiels et pédagogie de l'alternance des formations d'ingénieurs. Revue internationale de pédagogie de l'enseignement supérieur, 30(3).Repéré à http:// ripes.revues.org/872

Barge, S. (2010). Principles of problem and project based learning.Esbjerg et Copenhague : Aalborg University Press.

Bartel-Radic, A. (2009). La compétence interculturelle : état de l'art et perspectives. Management international, 13, 11-26.

Body Consortium (2013). Manuel des formateurs. Union Européenne / Grundtvig. Repéré le 27 mars 2015 à http://www.bodyproject.eu/media/BODY_Manual_FR.pdf

Byram, M. (2009). Sociétés multiculturelles et individus pluriculturels : le projet de l'éducation interculturelle. Strasbourg : Éditions du Conseil de l'Europe.

Byram, M., Gribkova, B. \& Starkey, H. (2002). Développer la dimension interculturelle dans l'enseignement des langues. Une introduction pratique à l'usage des enseignants. Strasbourg : Éditions du Conseil de l'Europe.

Camilleri, C. (1993). Les conditions structurelles de l'interculturel. Revue française de pédagogie, 103, 43-50.

Cazenave, C. (2003). La formation interculturelle. Paris : L'Harmattan.

CDIO (2001). Syllabus. A statement of goals for undergraduate engineering education.Repéré le 27 mars 2015 à http://www.cdio.org

Champagnol, R. (1974). Aperçu sur la pédagogie de l'apprentissage par résolution de problèmes. Revue française de pédagogie, 28, 21-27.

Chauvigné, C. \& Coulet, J. P. (2010). L'approche par compétences : un nouveau paradigme pour la pédagogie universitaire ? Revue française de pédagogie, 172, 15-28.

Clanet, C. (1993). Introduction aux approches interculturelles en éducation et en sciences humaines. Toulouse : PU du Mirail.

Clark, R. (2009). A PBL approach to teachning projet management. Dans Proceedings of the 2nd International Research Symposium on PBL, Melbourne, Australia.

Cohen-Emérique, M. (1993). L'approche interculturelle dans le processus d'aide. Santé mentale au Québec, 18(1), 71-91.

CTI (2009). Références et orientations. Repéré le 27 mars 2015 à http://www.cti-commission.fr/ References-et-Orientations-2009

Conseil de l'Europe (2008). Livre blanc sur le dialogue interculturel. Strasbourg : Éditions du Conseil de l'Europe.

Cuche, D. (1996). La notion de culture dans les sciences sociales. Paris : La Découverte.

Cuche, D. (1997). Nouveaux regards sur la culture. L'évolution d'une notion en anthropologie. Sciences Humaines, 77, 20-27.

Demorgon, J. (2003). L'interculturel entre réception et invention. Contextes, médias, concepts. Questions de communication, 4, 43-70.

Dervin, F. (2004). Définition et évaluation de la compétence interculturelle en contexte de mobilité : ouvertures. Moderna Språk, 66-76.

Dervin, F. (2011). Impostures interculturelles. Paris : L'Harmattan / Logiques sociales. 
Gadamer, H. G. (1976). Vérité et méthode. Paris : Seuil.

Giordan, A. \& De Vecchi, G. (1987). Les origines du savoir. Neuchatel : Delachaux.

Habermas, J. (1981). Théorie de l'agir communicationnel. Paris : Fayard.

Hameline, D. (1979). Les objectifs pédagogiques en formation initiale et en formation continue. Paris :

ESF.

Korhonen, K. (2003). Developing Intercultural Competence as Part of Professional Qualifications.

Communication présentée au 10th NIC Symposium on Intercultural Communication, Göteborg

University.

Lavanchy, A., Gajardo, A. et Dervin, F. (2011). Anthropologies de l'interculturalité. Paris :

L'Harmattan.

Leclerc, C., Bourassa \& B., Filteau, O. (2010). Utilisation de la méthode des incidents critiques dans une perspective d'explicitation, d'analyse critique et de transformation des pratiques professionnelles. Éducation et francophonie, 38(1), 11-32.

Lescarbeau, R. (2000). La méthode de l'incident critique. Interactions, 4(1), 159-164.

MEDEF (1998). Fiche glossaire. Dans Journées internationales de la formation. Objectif compétences.

Deauville.

Moeschler, J. \& Reboul, A. (1994). Dictionnaire encyclopédique de pragmatique. Paris : Seuil.

Neuner, G. (2003). Les mondes socioculturels intermédiaires dans l'enseignement et

l'apprentissage des langues vivantes Dans M. Byram (dir.), La compétence interculturelle (p. 15-66).

Strasbourg : Éditions du Conseil de l'Europe.

Pentecouteau, N. (2012). L'alternance dans une formation professionnelle universitaire. De l'idéal épistémologique aux contradictions pédagogiques. Revue internationale de pédagogie de l'enseignement supérieur, 28(1). Repéré à http://ripes.revues.org/605

Poirier Proulx, L. (1997). Enseigner et apprendre la résolution de problèmes. Pédagogie collégiale, 11(1), 18-23.

Romainville, M. (1999). L'apprentissage par problèmes. Réseau, 44, mars, SPU - FUNDP, Université de Namur.

Scallon, G. (2004). L'évaluation des compétences et l'importance du jugement. Pédagogie collégiale, 18(1), 14-20.

Stoiciu, G. (2008). L'émergence du domaine d'étude de la communication interculturelle. Hermès, $51,33-40$.

UNESCO (2013). Compétences interculturelles. Cadre conceptuel et opérationnel. Plate-forme intersectorielle pour une culture de la paix et de la non-violence.

Weisser, M. (2007). Méthodes d'analyse des interactions verbales au service d'une didactique comparée. Revue française de pédagogie, 158, 103-115.

Weisser, M. \& Rémigy, M.J. (2005). Argumenter en classe : à propos de quoi ? Comment ? Pourquoi ? L'Année de la Recherche en Sciences de l'Éducation, 129-148.

Wittorski, R. (2012). La professionnalisation de l'offre de formation universitaire : quelques spécificités. Revue internationale de pédagogie de l'enseignement supérieur, 28(1). Repéré à http:// ripes.revues.org/580 
Zarate, G. (2003). Identités et plurilinguisme : conditions préalables à la reconnaissance des compétences interculturelles. Dans M. Byram (dir.), La compétence interculturelle (p. 89-123). Strasbourg : Éditions du Conseil de l'Europe.

\section{NOTES}

1. Ce texte s'inscrit dans le cadre d'un projet de recherche financé par NovaTris, centre de compétences transfrontalières, IDEFI de l'Université de Haute-Alsace.

2. http://fond-documentaire.cti-commission.fr/fr/fond_documentaire/document/10/chapitreelement $/ 450$

3. Voir à ce sujet la taxonomie des niveaux de capacités socio-affectives de Krathwohl (1976, dans Hameline 1979, p. 128-129)

\section{RÉSUMÉS}

Dans une économie mondialisée, le métier d'ingénieur nécessite une ouverture aux phénomènes interculturels, tant dans leurs tâches de gestion de production que dans leurs relations professionnelles et commerciales internationales. Cet article a pour but de proposer quelques pistes pour former les compétences interculturelles nécessaires au sein des écoles d'ingénieurs. Nous commençons par analyser les liens entre culture et interculturalité. Nous montrons ainsi le rôle d'argument attribué aux traits culturels retenus dans une interaction langagière. Nous caractérisons ensuite des compétences interculturelles nécessaires aux ingénieurs, sensibles au contexte comme le veut la notion de compétence, en croisant les référentiels de formation habituels avec les réflexions du Conseil de l'Europe à ce sujet, et donc en en identifiant les ressources en termes de savoir-être, connaissances, savoir-comprendre, savoir apprendre et savoir s'engager. Ceci nous mène finalement à proposer deux méthodes de formation de ces compétences, qui tiennent compte à la fois des publics visés et des caractéristiques de la pédagogie universitaire : l'apprentissage par incidents critiques et par résolution de problèmes.

In a globalized economy, working as an engineer requires to be open to various intercultural phenomena as typically encountered through both production management tasks and international or business relationships. The purpose of this article is to expose ideas on how to provide training to these expected intercultural skills within the framework of engineering schools. At first, the link between culture and interculturality is investigated, showing the role of argument which is assigned to cultural features deriving from a language interaction. By combining French national training standards with the related discussions conducted by the European Council, the intercultural skills needed by tomorrow engineers are further characterized. Finally, two dedicated training methods are proposed, taking into account both the targeted audiences and the features of university pedagogy (i.e. critical incident learning and problem-based learning). 
INDEX

Mots-clés : apprentissage par incidents critiques, apprentissage par résolution de problèmes, compétences interculturelles, formation professionnelle, ingénieurs, pédagogie universitaire, référentiel de compétences

\section{AUTEUR}

\section{MARC WEISSER}

Université de Haute-Alsace, Mulhouse, France

Marc.Weisser@uha.fr 